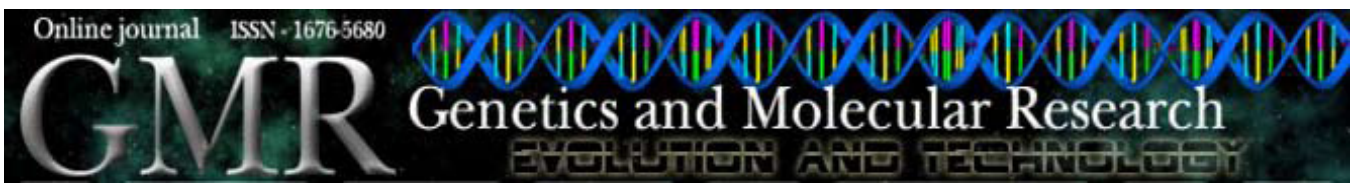

Thesis Abstract

\title{
Spermatogenesis and nucleolar behavior in Pentatomidae (Heteroptera) species of economic importance
}

\author{
H.V. Souza \\ 2008. UNESP - Universidade Estadual Paulista. Instituto de Biociências, \\ Letras e Ciências Exatas, São José do Rio Preto, SP, Brasil. Master's thesis. \\ Orienting professor: Prof. Dr. Mary Massumi Itoyama
}

Heteroptera are the most important group of insects of economic importance, because most of them feed on crops or stored grains used for human consumption. The cytogenetic analyses showed that Antiteuchus tripterus has a compound lobe (5/6), which could be involved in the formation of the harlequin lobe, giving evidence that evolutionary pressure existed for the maintenance of this lobe in this species, which demonstrates the importance of this lobe for some species. All species analyzed have XY sexual chromosome systems and a chromosome complement of $2 \mathrm{n}=14$ chromosomes. Furthermore, the analyses of nucleolar behavior showed that there are different metabolic activities in lobes 4, 5, and 6 of A. tripterus and in the species without the harlequin lobe, such as Platycarenus umbractulatus, that also had differential marking of lobe 5 , which could indicate the formation of non-fertilizing sperm. The species Euschistus heros did not have the harlequin lobe; however, analyses of spermatogenesis showed that there are significant differences in cell size in lobes 4 and 6 , which may be related to the formation of non-fertilizing sperm. Nucleolar bodies with "mushroom-like" morphology were found in the species of the genus Oebalus, a previously undescribed feature. Moreover, two dark and two light bodies were observed in P. umbractulatus, which may be due to the participation of sex chromosomes in the formation and organization of the nucleolus in this species. We conclude that such analyses are important for understanding the evolution of the Pentatomidae. 